\title{
Mutation profiles of follicular thyroid tumors by targeted sequencing
}

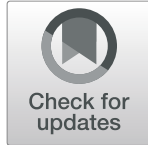

Huanli Duan, Xiaoding Liu, Xinyu Ren, Hui Zhang, Huanwen Wu* and Zhiyong Liang*

\begin{abstract}
Background: One of the major challenges remaining in the classification of thyroid tumor is the determination of whether a nodule is benign or malignant. We aimed to characterize the mutational profiles of follicular thyroid tumor and to identify markers with potential diagnostic and prognostic implications.

Methods: Targeted sequencing with a panel of 18 thyroid cancer-related genes was performed on 48 tissue samples from follicular thyroid adenoma (FTA), 32 follicular tumors of uncertain malignant potential (FT-UMP), 17 welldifferentiated tumors of uncertain malignant potential (WDT-UMP) and 53 samples from follicular thyroid carcinoma (FTC). The correlation of mutation profiles and clinicopathological features and prognosis were also analyzed.

Results: We identified 95 nonsilent mutations spanning 14 genes. Specifically, TERT promoter (TERTp) mutations were exclusively detected in FTC. A total of 80\% EIF1AX exon 2 mutations (4/5) and 75\% TSHR mutations (3/4) occurred in FTA, whereas the rest of them occurred in FT-UMP. KRAS mutations and TP53 mutations were only presented in borderline or malignant tumors. H/N-RAS mutations were detected in all four subtypes, but were most commonly found in WDT-UMP $(p=0.031)$. All N-RAS mutations were located at codon 61. BRAF V600E and RET fusion were absent in the entire cohort. In FTC cases, EIFIAX mutations were all located at intron 5/exon 6 and correlated with advanced disease $(p=0.032)$. Both EIFIAX and TERTp mutations predicted shorter disease-free survival $(p=0.007, p=0.024$, respectively). Further analysis revealed that TERTp mutations were correlated with shorter disease-free survival in patients with minimally invasive /encapsulated angioinvasive FTC $(p=0.017)$, but not in those with widely invasive FTC $(p=0.297)$.
\end{abstract}

Conclusion: TERTp, EIFIAX, TSHR, H/N/K-RAS and TP53 mutations may have diagnostic or prognostic potential in follicular thyroid tumors. TERTp mutations may predict a poor outcome in patients with minimally invasive/ encapsulated angioinvasive FTC.

Keywords: Follicular thyroid tumor, Targeted next generation sequencing, TERT promoter mutation

\section{Background}

Thyroid cancer, the most common type of endocrine malignancy, predominantly arises from thyroid follicular cells, with approximately $95 \%$ being differentiated thyroid cancer. As one of the categories, follicular thyroid carcinoma (FTC) is a high-risk cancer with the likelihood of distant relapse [1].FTC is the malignant counterpart of follicular thyroid adenoma (FTA), the latter commonly found as benign neoplasm of the thyroid gland. Thyroid tumors with uncertain malignant potential (TT-UMP) are defined as the tumors presenting

\footnotetext{
*Correspondence: whw14093@163.com; liangzhiyong1220@yahoo.com Molecular Pathology Research Center, Department of Pathology, Peking Union Medical College Hospital, Chinese Academy of Medical Science, Peking Union Medical College, Beijing 100730, China
}

questionable capsular or vascular invasion and fail to meet the criteria for carcinoma, comprising follicular tumors with uncertain malignant nature (FT-UMP) and well-differentiated tumor of uncertain malignant potential (WDT-UMP) [2]. Currently, one of the major challenges remaining in the differentiation among FTC, TT-UMP and FTA is the interobserver variability in the histologic interpretation of capsular or vascular invasion, which largely depends on the liberty of pathologists, even in the surgically resected samples [3, 4].

TERT promoter (TERTp) mutations, firstly reported in both familial and sporadic melanomas, were regarded as important mechanisms contributing to increased telomerase activity in malignant cells [5]. Recent studies

(c) The Author(s). 2019 Open Access This article is distributed under the terms of the Creative Commons Attribution 4.0 International License (http://creativecommons.org/licenses/by/4.0/), which permits unrestricted use, distribution, and 
showed that in FTC, the frequency of TERTp mutations ranges from 14 to $36 \%$ [6-12]. Numerous studies have shown that TERTp mutations were more popular in advanced thyroid cancers and strongly correlated with poor clinical outcomes [6-8, 13]. EIF1AX gene was recently identified as a new thyroid cancer-related gene. EIF1AX mutations were strikingly enriched in poorly differentiated thyroid cancer (PDTC) and anaplastic thyroid cancer (ATC) and predicted for shorter survival in PDTC [14]. Distinct mutations on EIF1AX may be correlated with different tumor phenotypes [15]. However, the role of TERTP or EIF1AX mutations in follicular thyroid tumors hadn't been fully investigated.

With the development of high-throughput sequencing technologies, next- generation sequencing (NGS)-based molecular testing is playing a vital role in diagnosis, prognosis and treatment monitoring [16]. For example, the identification of $B R A F$ V600E from fine-needle aspiration specimens is used to diagnose papillary thyroid cancer (PTC) or PTC-derived anaplastic thyroid cancer [17]. The purpose of the study was to derive mutation profiles in a representative cohort of patients with FTA/FT-UMP/WDT-UMP/FTC using capture-based targeted sequencing with an 18-gene panel and to correlate the mutation profiles with clinicopathological features and prognosis.

\section{Materials and methods Study cases}

One hundred and fifty-three cases initially diagnosed as FTA, atypical follicular thyroid adenoma (AFTA) and FTC between June 2010 and May 2015 were derived from the database of the Department of Pathology, Peking Union Medical College Hospital. All patients received surgical treatment. All corresponding archived hematoxylin and eosin-stained tumor sections were collected and independently re-evaluated by two experienced pathologists (Wu $\mathrm{H}$ and Ren $\mathrm{X}$ ). The third pathologist (Liang Z) was available if necessary. Finally, 152 cases including 48 FTA, 17 WDT-UMP, 32 FT-UMP and 55 FTC were histologically verified based on the 2017 World Health Organization (WHO) classification of endocrine tumors. FTC was further classified into minimally invasive group, encapsulated angioinvasive group and widely invasive group. Hürthle cell tumors were integrated into subtypes of those tumors, although they were suggested as distinctive ones [2]. The American Joint Committee on Cancer (AJCC) stages were defined according to the 8th edition. Moreover, patient demographics and survival outcomes were obtained. The institutional review board of Peking Union Medical College Hospital approved the study, and because of its retrospective nature, written informed consent was waived.

\section{DNA extraction}

Ten tumor sections (5 $\mu \mathrm{m}$ thick each) from qualified formalin-fixed, paraffin-embedded (FFPE) tissue blocks were mounted on slides. The tumor sections were deparaffinized using xylene, dehydrated step-wise with ethanol, and one of the slides stained with HE. An experienced pathologist (Wu H) examined and marked the target area of tumor cells on the slides. DNA was extracted using QIAamp DNA FFPE Tissue Kit (QIAGEN, California, USA) according to the manufacturer's instructions. Quality control of DNA samples were evaluated using the Invitrogen Qubit 3 fluorometer (Thermo Fisher Scientific, California, USA).

\section{Library preparation}

DNA fragmentation was performed using Covaris M220, followed by end repair, phosphorylation and adaptor ligation. Fragments of size 200-400 bp were selected by AMPure beads (Agencourt AMPure XP Kit, Beckman Coulter, California, USA) followed by hybridization with capture probes baits, hybrid selection with magnetic beads and PCR amplification. Subsequently, high-sensitivity DNA assay was performed to assess the quality and the size of all fragments. Indexed samples were sequenced on Nextseq500 sequencer (Illumina, Inc., California, USA) with paired-end reads using 18 thyroid cancer-related genes. The panel comprises 18 genes, which are closely relevant to the pathogenesis and development of thyroid cancer. The panel covers selected exons, introns or promoter regions of TERT, EIF1AX, H/N/K-RAS, BRAF, TP53, PIK3CA, PTEN, GNAS, TSHR, CTNNB1, AKT1 and ETV6, and was designed to detect single nucleotide substitutions and small indels in all these genes. Additionally, the panel was capable of identifying large gene rearrangements at RET, PPARG, $A L K$, and NTRK1. Both known hotspot mutations and novel variants could be detected.

\section{NGS analysis}

Sequencing data was mapped to the human genome (hg19) using Burrows-Wheeler Aligner 0.7.10. Local alignment optimization, variant calling and annotation were performed using GATK 3.2, MuTect, and VarScan. DNA translocation analysis was performed using both Tophat2 and Factera 1.4.3. Variants were filtered using the VarScan filter pipeline. According to the ExAC, 1000 Genomes, dbSNP, ESP6500SI-V2 database, variants with population frequency over $0.1 \%$ were grouped as common SNPs and removed. Remaining variants were annotated with ANNOVAR and SnpEff v3.6. To avoid errors related to sequencing or aligning, variants were filtered to retain only those covered by at least 100 reads, and Integrative Genomics Viewer (Broad Institute, USA) was employed to visualize variants aligned against the reference genome to confirm the accuracy of the variant calls 
by checking for possible strand biased and sequencing errors.

All variants were either confirmed somatic ones (as described in the Catalogue Of Somatic Mutations In Cancer (COSMIC) database [18]) or were predicted by three algorithms including SIFT, PolyPhen-2 and PROVEAN [19]. Mutations likely to result in altered protein function were described as deleterious, damaging, or probably damaging. Mutations shown simultaneously as tolerated, benign and neutral in the three algorithms, or mutations predicted as neutral in COSMIC database were excluded from further analysis. H/N/K-RAS, PTEN, RET, BRAF, EIF1AX and TSHR are known as early driver genes in thyroid carcinogenesis, and when the allelic frequency was $\geq 10 \%$, the mutations were regarded as positive test results. Mutations in genes, such as TP53, TERT, CTNNB1 and PIK3CA are developed subsequently in the process of carcinogenesis, and mutations were considered as positive ones if their allelic frequency was $\geq 5 \%[20]$.

\section{Statistical analyses}

Data was presented either as frequencies and percentages, or as means and standard deviations or medians and interquartile range. Categorical variables were compared using either Pearson chi-square test or Fisher's exact test. Continuous variables were compared using either the independent $t$ test or one-way analysis of variance. Disease-free survival (DFS) was determined between the date of evaluation, and the date of recurrence or date of last known status. Survival curves were plotted with Kaplan-Meier method with log-rank statistics. Cox proportional hazards regression was used to assess the risk of recurrence. Statistical significance was defined as two-sided values of $P<0.05$. Statistical analyses were conducted with SPSS version 23.0 (SPSS Inc). Mutation plots were generated using Mutation Mapper tools, which are available at the cBioPortal [21, 22]. Graphic representations of gene mutations were performed on GraphPad Prism 7.00 (GraphPad Software).

\section{Results}

Mutation signature for distinguishing FTA, TT-UMP and FTC We profiled 150 patients with follicular thyroid tumors, including 48 with FTA, 32 with FT-UMP, 17 with WDT-UMP and 53 with FTC (Two cases of FTC were deleted due to DNA sequencing failure) (Table 1). A total of $49 \%$ of patients (73/150) carried at least one mutation, involving 95 nonsilent somatic mutations spanning 14 genes (Fig. 1). Genetic alterations were summarized and compared. First, the rates of concurrent mutations were different among the four subtypes. Approximately, 2\% FTA (1/48), 6\% WDT-UMP (1/ 17), 13\% FT-UMP (4/32) and 21\% FTC cases (11/53) carried concurrent gene mutations. Three concurrent somatic mutations were only detected in patients with FTC $(8 \%, 4 /$ 53). There was a significant difference about the rates of concurrent mutations in at least two genes among the four subtypes $(p=0.018) . H / N / K-R A S$ were the most frequently co-mutated genes. Second, the predominant gene mutation feature was different. TERTp mutations were exclusively detected in FTC. A total of $80 \%$ EIF1AX exon 2 mutations (4/ 5 ) and $75 \%$ TSHR mutations (3/4) occurred in FTA, whereas 20\% EIF1AX exon 2 mutations (1/5) and 25\% TSHR mutations $(1 / 4)$ occurred in FT-UMP. KRAS mutations and TP53 mutations were only present in the borderline or malignant tumors. H/N-RAS mutations were detected in all four subtypes, but were most commonly found in WDT-UMP $(p=0.031)$. BRAF V600E and RET fusions were absent in the entire cohort. Overall, TERTp, EIF1AX, TSHR, H/N/K-RAS and TP53 genes may be helpful to differentiate the subtypes of follicular thyroid tumors.

\section{H/N/K-RAS, TERTp and EIF1AX mutations in follicular thyroid tumors}

We identified frequent $H / N / K-R A S$ mutations in all subtypes $(28 \%, 42 / 150)$. HRAS mutations were detected in $8 \%$ FTA, 12\% WDT-UMP, 3\% FT-UMP and 8\% FTC cases. They were located at codon 61/13. NRAS mutations were identified in 6\% FTA, 35\% WDT-UMP, 16\% FT-UMP and $19 \%$ FTC cases. They were all located at codon 61. KRAS mutations were found in 6\% WDT-UMP, 9\% FT-UMP and 6\% FTC. They were mainly located at codon 61 (57\%, 4/7). H/N/K-RAS genes were mutated exclusively except for one case of FT-UMP exhibiting concurrent KRAS and NRAS mutations. Our results showed TERTp mutations were exclusively detected in $25 \%$ of patients with FTC (13/53). C228T was the predominant form of mutation, accounting for $92 \%$ of detected mutations (12/13) and the other was C250T. EIF1AX mutations were observed in FTC, FT-UMP and FTA. EIF1AX mutations were identified in 6\% FTC cases (3/53), and all located at intron 5/ exon 6 (2 EIF1AX A113_splice and 1 G124* mutation). Two of three EIF1AX mutations were concurrent with NRAS mutations. EIF1AX mutation were identified in $13 \%$ FTA (6/48), 4 EIF1AX exon 2 and 2 A113_splice mutations. One EIF1AX A113_splice mutation coexisted with HRAS mutation. More details were shown in Fig. 2. In order to have an overall understanding of EIF1AX mutation detected in thyroid tumors, we also analyzed the occurrence of EIF1AX mutations in the COSMIC database [23], cBioPortal for cancer genomics database [24] and previous studies [14, 15, 25-29] (Table 2). EIF1AX intron5/exon6 mutations occurred in benign or malignant thyroid tumors. Concurrent EIF1AX exon 2 and $H / N /$ $K-R A S$ mutations were only observed in PDTC and ATC. EIF1AX exon 2 mutations without $H / N / K-R A S$ mutations were only detected in the benign lesions, borderline lesions and PTC. 
Table 1 Clinicopathological features of 150 patients with follicular thyroid tumors

\begin{tabular}{|c|c|c|c|c|}
\hline & FTC, n (\%) & FTA, n (\%) & FT-UMP, n (\%) & WDT-UMP, n(\%) \\
\hline Cases & 53 & 48 & 32 & 17 \\
\hline \multicolumn{5}{|l|}{ Sex } \\
\hline Male & $16(30)$ & $16(33)$ & $12(37)$ & $5(29)$ \\
\hline Female & $37(70)$ & $32(67)$ & $20(63)$ & $12(71)$ \\
\hline \multicolumn{5}{|l|}{ Age at diagnosis } \\
\hline Mean year & 46 & 45 & 46 & 44 \\
\hline Median (Quartiles) & $50(31-61)$ & $47(34-54)$ & $44(37-58)$ & $43(33-55)$ \\
\hline \multicolumn{5}{|l|}{ Tumor size, $\mathrm{cm}$} \\
\hline Mean size & 3.1 & 2.5 & 3.4 & 1.9 \\
\hline Median (Quartiles) & $2.5(1.5-4.0)$ & $2.5(1.8-3.0)$ & $2.5(1.4-5.4)$ & $1.2(1.0-2.7)$ \\
\hline \multicolumn{5}{|l|}{ Variant type } \\
\hline Hürthle cell tumor & $14(26)$ & $12(25)$ & $6(19)$ & $2(18)$ \\
\hline Non-Hürthle cell tumor & $39(74)$ & $36(75)$ & $26(81)$ & $14(82)$ \\
\hline \multicolumn{5}{|l|}{ Histologic type ${ }^{a}$} \\
\hline Minimally invasive & $25(47)$ & & & \\
\hline Encapsulated angioinvasive & $15(28)$ & & & \\
\hline Widely invasive & $13(25)$ & & & \\
\hline \multicolumn{5}{|l|}{ Lymph node metastasis } \\
\hline Present & $5(9)$ & - & - & - \\
\hline \multicolumn{5}{|l|}{ Distant metastasis } \\
\hline Present & $2(4)$ & - & - & - \\
\hline \multicolumn{5}{|l|}{ AJCC stage } \\
\hline $\mid \sim \|$ & $51(96)$ & - & - & - \\
\hline\|\|$\sim \mid V$ & $2(4)$ & - & - & - \\
\hline \multicolumn{5}{|l|}{ Follow-up time } \\
\hline Median (Quartiles) & $56(46-65)$ & $55(42-65)$ & $55(47-60)$ & $55(29-74)$ \\
\hline \multicolumn{5}{|l|}{ Disease Persistence/Recurrence } \\
\hline Yes & $8(15)$ & $0(0)$ & $0(0)$ & $0(0)$ \\
\hline No & $45(85)$ & $48(100)$ & $32(100)$ & $16(100)$ \\
\hline
\end{tabular}

a , based on the 2017 World Health Organization classification of endocrine tumors; Abbreviations: FTC follicular thyroid carcinoma, FTA follicular thyroid adenoma, FT-UMP follicular tumor of uncertain malignant potential, WDT-UMP well-differentiated tumor of uncertain malignant potential, AJCC American Joint Committee on Cancer, 8th edition staging;

In FTC, we illustrated that patients with co-occurring TERTp and $H / N / K-R A S$ mutations were older and presented with advanced disease $(p=0.013, p=0.015$, respectively), compared to other patients (Table 3). Similarly, we clarified EIF1AX mutations were correlated with advanced disease $(p=0.032)$, with $2 \%$ of mutation rate in stage I FTC (1/44), $14 \%$ of mutation rate in stage II FTC (1/7), 100\% of mutation rate in stage III FTC (1/1).

\section{Genetic comparison of Hürthle cell tumors and non- Hürthle cell tumors}

Hürthle cell tumors were separated from the follicular tumors for their distinct genetic profiles in the 2017 WHO classification of endocrine tumors. In our cohort, 35 Hürthle cell tumors and 115 non-Hürthle cell tumors cases were identified. Genetic comparison of the two types of thyroid tumors were performed. First, TERTp and KRAS mutations were identified in 17 and $11 \%$ Hürthle cell tumors, respectively, whereas it was 6 and $3 \%$ in non-Hürthle cell tumors, respectively. Second, NRAS mutations tended to be more popular in non-Hürthle cell tumors with incidence of $19 \%$, compared with that of $6 \%$ in Hürthle cell tumors. Six co-occurring TERTp and H/N-RAS mutations all occurred in non-Hürthle cell tumor, whereas no such alteration was observed in Hürthle cell tumor.

\section{Survival analysis}

In our cohort, survival outcomes could be evaluated in 94\% FTA (45/48), 100\%WDT-UMP (17/17), 91\% FT-UMP 


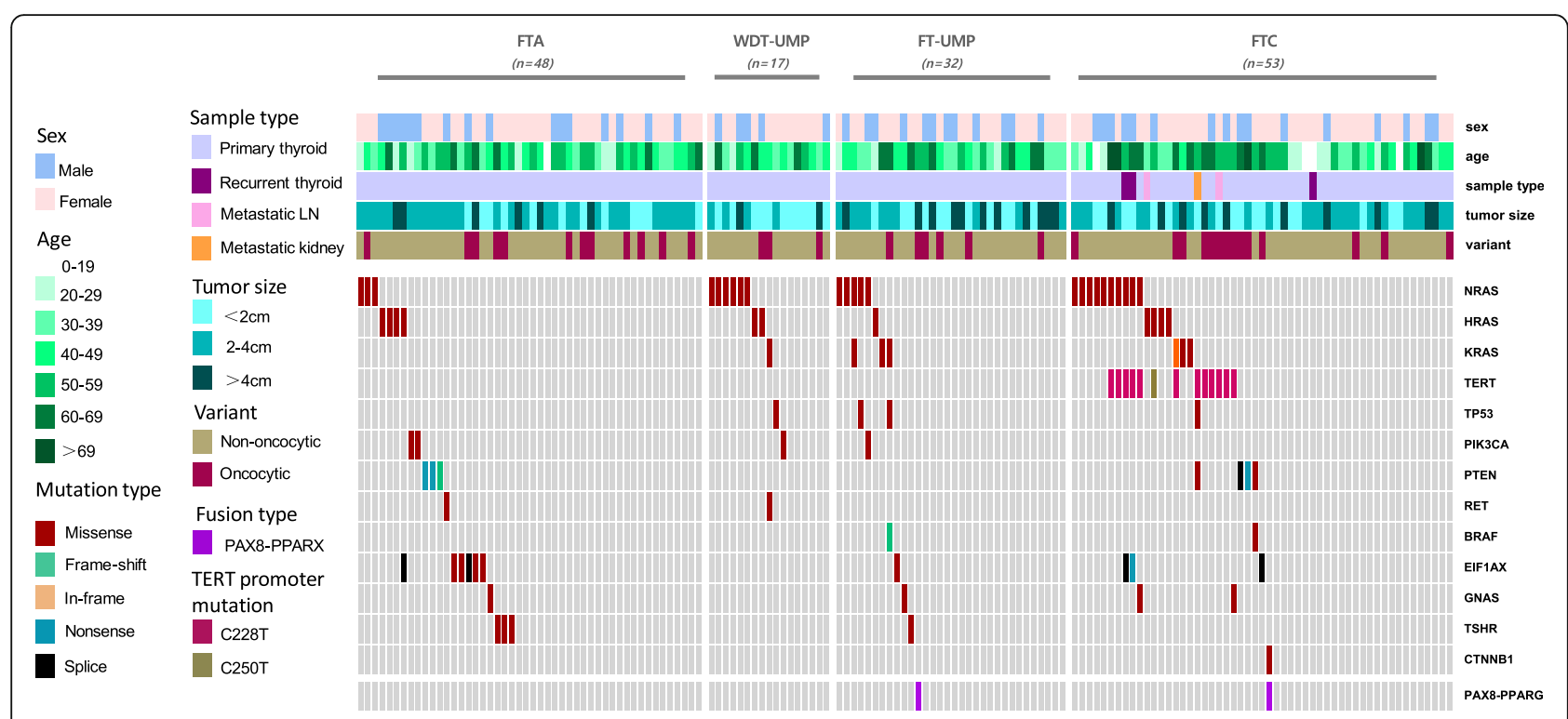

Fig. 1 Clinicopathological features and mutation spectrum in 150 follicular thyroid tumors. Clinicopathological characteristics and genes were listed on the right of the mutation spectrum and color keys for them were shown on the left

(29/32) and 98\%FTC (52/53). Only one FTC patient with initial bone metastasis maintained disease persistence and experienced disease-specific death. Another FTC patient with kidney metastasis at diagnosis was lost to follow-up. Collectively, seven patients with FTC exhibited disease recurrence in the first four years, comprising four local recurrence, one reginal recurrence and two distant recurrences (both spread to lungs). In contrast, no disease recurrence was observed in FTA, FT-UMP or WDT-UMP patients. We performed a detailed investigation regarding the correlation of clinicopathological and genetic variables and disease-free survival (Table 4). Based on the univariate Cox analysis, predictors of shorter DFS were lymph node metastasis (95\% confidence interval (CI) 1.929$39.145, p=0.005)$ advanced disease (95\% CI 1.646$152.169 ; p=0.017$ ), widely invasive histologic subtype (95\% CI 1.084-21.761, $p=0.039$ ), EIF1AX mutations (95\% CI 1.887-52.892; $p=0.007$ ), and TERTp mutations $(95 \%$ CI $1.254-25.298 ; p=0.024)$. In multivariable analysis, no independent factors were identified.
We further analyzed the impact of TERTp mutation status on survival outcome in FTC patients with three histologic types. In patients with minimally invasive/encapsulated angioinvasive FTC, TERTp mutations contributed to shorter DFS than TERTp wild-type did $(p=0.017)$, whereas TERTp mutations were not correlated with shorter DFS in patients with widely invasive $\operatorname{FTC}(p=0.297)$. Kaplan-Meier survival analysis and compared statistically using the log-rank test were performed (Fig. 3).

\section{Discussion}

FTA, FT-UMP, WDT-UMP and FTC can hardly be precisely distinguished based on histopathological features, and the differential diagnosis may sometimes be arbitrary and difficult. Molecular diagnostic tools would may help to determine accurate diagnosis by exploring the genetic characteristics. Our study used capture-based targeted sequencing with an 18-gene thyroid tumor panel to detect and quantify genetic alterations associated with these tumors, aiming to derive potential diagnostic and prognostic

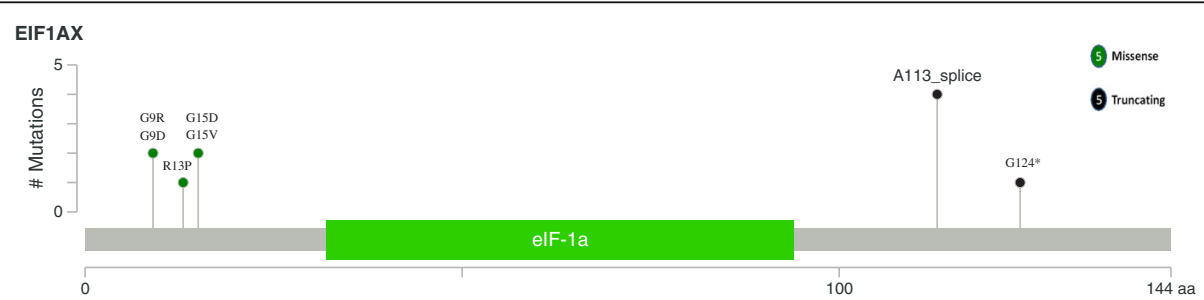

Fig. 2 EIFIAX mutations detected in our cohort. The colored box depicts the functional domain along the protein. The location of the circle specifies the mutation site. A patient is represented by a circle. Green circles represent missense mutations and black circles represent truncating mutations. The number of circles in a lollipop represents the number of patients harboring the specific variant 
Table 2 EIFIAX mutations were summarized based on our results, COSMIC database, cBioPortal for cancer genomics database and previous studies (references14-15, 23-27)

\begin{tabular}{|c|c|c|c|c|c|}
\hline \multirow[t]{2}{*}{ Disease } & \multirow[t]{2}{*}{ Cases } & \multicolumn{2}{|c|}{ EIF1AX exon2 mutations } & \multicolumn{2}{|c|}{ EIF1AX intron5/exon6 mutations ${ }^{a}$} \\
\hline & & Total cases & With RAS Mut & Total cases & With RAS Mut \\
\hline$\overline{\mathrm{FA} / \mathrm{HN}}$ & 15 & 10 & 0 & 5 & 2 \\
\hline FT-UMP & 1 & 1 & 0 & 0 & 0 \\
\hline PTC & 10 & 4 & 0 & 6 & 4 \\
\hline FTC & 7 & 0 & 0 & 7 & 4 \\
\hline PDTC & 13 & 3 & 3 & 10 & 8 \\
\hline ATC & 14 & 5 & 5 & 9 & 9 \\
\hline
\end{tabular}

a, A113_splice mutation and G124* mutations

Abbreviations: FA/HN follicular adenoma/hyperplastic nodules, FT-UMP follicular tumor with uncertain malignant potential, PTC papillary thyroid carcinoma, FTC follicular thyroid carcinoma, PDTC poorly differentiated thyroid carcinoma, ATC anaplastic thyroid carcinoma

Table 3 Association of TERTp and H/N/K-RAS mutations with clinicopathological featurs in 53 FTC patients

\begin{tabular}{|c|c|c|c|c|c|}
\hline Variations & H/N/K-RAS- TERTp- & H/N/K-RAS+ TERTp- & H/N/K-RAS- TERTp+ & H/N/K-RAS+ TERTp+ & $P$ \\
\hline Cases & 30 & 10 & 6 & 7 & \\
\hline Sex & & & & & 0.406 \\
\hline Male & $8(27)$ & $2(20)$ & $2(33)$ & $4(57)$ & \\
\hline Female & $22(73)$ & $8(80)$ & $4(67)$ & $3(43)$ & \\
\hline Age, years & & & & & 0.013 \\
\hline Median \pm SD & $43 \pm 3$ & $33 \pm 5$ & $59 \pm 2$ & $66 \pm 3$ & \\
\hline Tumor size $(\mathrm{cm})$ & & & & & 0.764 \\
\hline$<2.0$ & $8(27)$ & $3(30)$ & $3(50)$ & $3(43)$ & \\
\hline $2.0-4.0$ & $16(53)$ & $5(50)$ & $1(17)$ & $3(43)$ & \\
\hline$>4.0$ & $6(20)$ & $2(20)$ & $2(33)$ & $1(14)$ & \\
\hline Lymph node metastasis & & & & & 0.271 \\
\hline Yes & $2(7)$ & $1(10)$ & 0 & $2(29)$ & \\
\hline No & $28(93)$ & $9(90)$ & $6(100)$ & $5(71)$ & \\
\hline Distant metastasis & & & & & 0.057 \\
\hline Yes & 0 & 0 & $1(17)$ & $1(14)$ & \\
\hline No & $30(100)$ & $10(100)$ & $5(83)$ & $6(86)$ & \\
\hline AJCC Stage & & & & & 0.015 \\
\hline I & $27(90)$ & $10(100)$ & $4(67)$ & $3(43)$ & \\
\hline$\|$ & $3(10)$ & 0 & $2(33)$ & $2(29)$ & \\
\hline III & 0 & 0 & 0 & $1(14)$ & \\
\hline IV & 0 & 0 & 0 & $1(14)$ & \\
\hline Histologic type & & & & & 0.681 \\
\hline Minimally invasive & $16(53)$ & $5(50)$ & $2(33)$ & $2(28)$ & \\
\hline Encapsulated angioinvasive & $7(23)$ & $4(40)$ & $2(33)$ & $2(28)$ & \\
\hline Widely invasive & $7(23)$ & $1(10)$ & $2(33)$ & $3(43)$ & \\
\hline Disease recurrence/persistence ${ }^{a}$ & & & & & 0.026 \\
\hline Yes & $2(7)$ & $1(10)$ & $2(29)$ & $3(50)$ & \\
\hline No & $28(93)$ & $9(90)$ & $4(71)$ & $3(50)$ & \\
\hline
\end{tabular}

${ }^{a}$, fifty-two patients were available to evaluate the disease recurrence/persistence status, the other patient was lost to follow-up Abbreviations: TERTp, TERT promoter, FTC follicular thyroid carcinoma, AJCC American Joint Committee on Cancer, 8th edition staging, $P$ P-value, median \pm SD median \pm standard deviation 
Table 4 Association of clinicopathological and genetic characteristics with disease- free survival in 51 FTC

\begin{tabular}{|c|c|c|c|c|c|}
\hline \multirow[b]{2}{*}{ Variations } & \multirow[b]{2}{*}{ Cases } & \multicolumn{2}{|l|}{ Univariate } & \multicolumn{2}{|l|}{ Multivariate } \\
\hline & & $\mathrm{HR}(95 \% \mathrm{Cl})$ & $P$ & $\mathrm{HR}(95 \% \mathrm{Cl})$ & $P$ \\
\hline Sex & & & 0.138 & & \\
\hline Male & 16 & 1.000(reference) & & & \\
\hline Female & 35 & $0.321(0.072-1.439)$ & & & \\
\hline Age at diagnosis, years & & & 0.425 & & \\
\hline$<55$ & 29 & 1.000(reference) & & & \\
\hline$\geq 55$ & 22 & $1.840(0.411-8.234)$ & & & \\
\hline Tumor size, $\mathrm{cm}$ & & & 0.762 & & \\
\hline$<2.0$ & 16 & 1.000(reference) & & & \\
\hline $2.0 \sim 4.0$ & 24 & $0.637(0.128-3.158)$ & & & \\
\hline$>4.0$ & 11 & $0.471(0.049-4.531)$ & & & \\
\hline Lymph node metastasis & & & 0.005 & & 0.442 \\
\hline No & 46 & 1.000(reference) & & 1.000 (reference) & \\
\hline Yes & 5 & 8.689 (1.929-39.145) & & $2.632(0.224-30.951)$ & \\
\hline AJCC Stage & & & 0.017 & & 0.770 \\
\hline $1+\|$ & 50 & 1.000(reference) & & 1.000(reference) & \\
\hline$I I I+I V$ & 1 & $15.827(1.646-152.169)$ & & $1.742(0.043-71.363)$ & \\
\hline Histologic type ${ }^{a}$ & & & 0.039 & & 0.182 \\
\hline Minimally invasive /Encapsulated angioinvasive & 39 & 1.000 (reference) & & 1.000 (reference) & \\
\hline Widely invasive & 12 & $4.856(1.084-21.761)$ & & $4.125(0.515-33.049)$ & \\
\hline Hürthle cell tumors & & & 0.992 & & \\
\hline No & 37 & 1.000(reference) & & & \\
\hline Yes & 14 & $0.991(0.192-5.111)$ & & & \\
\hline EIF1AX mutation & & & 0.007 & & 0.366 \\
\hline WT & 48 & 1.000(reference) & & 1.000 (reference) & \\
\hline Mut & 3 & $9.989(1.887-52.892)$ & & $3.998(0.198-80.778)$ & \\
\hline H/N/K-RAS mutation & & & 0.393 & & \\
\hline WT & 44 & 1.000 (reference) & & & \\
\hline Mut & 7 & $1.920(0.430-8.586)$ & & & \\
\hline TERTp mutation & & & 0.024 & & 0.162 \\
\hline WT & 40 & 1.000 (reference) & & 1.000 (reference) & \\
\hline Mut & 11 & $5.633(1.254-25.298)$ & & $3.841(0.583-25.307)$ & \\
\hline
\end{tabular}

abased on the 2017 World Health Organization classification of endocrine tumors; Abbreviations: WT wild-type, FTC follicular thyroid carcinoma, AJCC American Joint Committee on Cancer, 8th edition staging, $H R$ hazard ratio, 95\%Cl 95\% confidence interval, $P$ P-value

biomarkers. Our results showed that TERTp, EIF1AX, TSHR, H/N/K-RAS and TP53 genes may be potential diagnostic or prognostic markers in follicular thyroid tumors. In FTC, TERTp mutations were correlated with shorter DFS in patients with minimally invasive/encapsulated angioinvasive histologic feature, but not in patients with widely invasive histologic feature.

Given that the molecular similarities concerning TERTP mutations or aberrations between FTC and FT-UMP groups, and that the TERTp-mutated FTA developed a scar recurrence and died of FTC, TERTp mutational testing is thought to detect malignant potential in follicular thyroid tumors [6, 11]. Our results showed patients with FT-UMP or FTA carried no TERTp mutations, in agreement with previous studies, which reported that normal tissues and benign lesions of the thyroid carried no TERTp mutations [7-10, 12, 13]. The reasons may be due to the rarity of TERTp mutations in non-malignant follicular thyroid tumors, inadequate tumor sampling or interobserver variability between pathologists in diagnosing thyroid borderline tumors $[3,26,30]$. The interobserver variability may be affected by the differences in clinical practice in the different regions. Borderline tumors were often treated as thyroid 


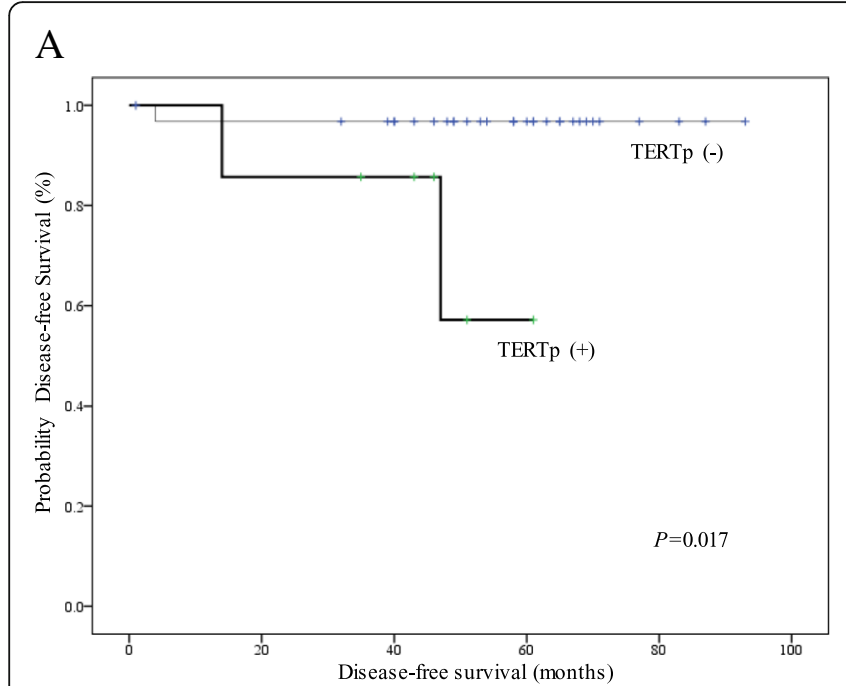

B

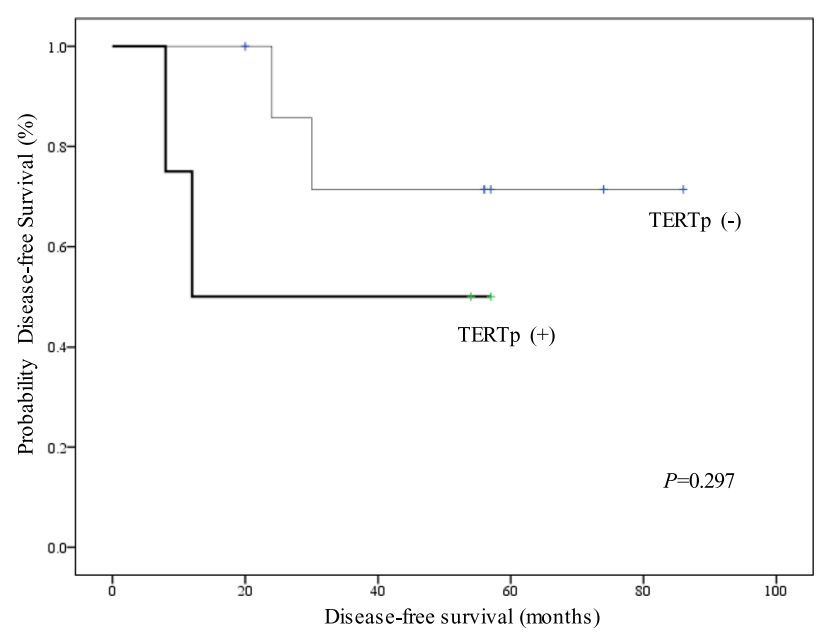

Fig. 3 Kaplan-Meier curves of disease-free survival by TERTp mutational status in patients with FTC. Results from the analysis of patients with (a) minimally invasive /encapsulated angioinvasive histologic type and (b) widely invasive histologic type

carcinoma in western clinical practices, whereas according to Asian practice they were handled as if benign tumors [30]. Additional international cooperation might be needed to further clarify the role of TERTp mutations in FTC tumorigenesis.

Interestingly, our further analysis showed that TERTp mutations were significantly correlated with shorter DFS in patients with minimally invasive/encapsulated angioinvasive FTC, but not in patients with widely invasive FTC. Recently, Bournaud et al. also reported that TERTp mutations might be helpful to better define the prognosis of localized thyroid cancer without aggressive histology. However, only a few cases of FTC were included in their study [31]. It provides a new perspective on the prognostic value of TERTp mutations in FTC. We also clarified that FTC patients with co-occurring TERTp and H/N/K-RAS mutations were older, presented with advanced disease, and had higher disease recurrent/persistent rate, compared with other FTC patients. Moreover, the only deceased patient in our cohort was the patient with FTC carrying concurrent TERTp and NRAS mutations. Therefore, the synergistic role of TERTp and H/N/K-RAS mutations might exist in FTC, which is in agreement with literature findings $[13,32,33]$.

Our results showed that NRAS mutations were more detected in non-Hürthle cell tumors, whereas KRAS mutations were more common in Hürthle cell tumors. That is in accordance with the study by Radkay et al. [34], where compared to $H / N-R A S$ mutation, KRAS mutation was more common in thyroid nodules with oncocytic change. Coexisting TERTp and H/N-RAS mutations were all detected in non-Hürthle cell cancer, whereas TERTp without $H / N-R A S$ mutations occurred in Hürthle cell cancer. These results indicated biologically distinct genetic features between non-Hürthle and Hürthle cell tumors.

Co-occurrence of EIF1AX and H/N/K-RAS mutations is correlated with tumor aggressiveness, especially when it is the A113_splice mutations for EIF1AX gene [15, 25]. That is consistent with our results that EIF1AX mutations in FTC were located at intron 5/exon 6, correlated with advanced disease, and coexisted with NRAS mutation. Moreover, we also identified one EIF1AX G124* nonsense mutation in FTC, which was only previously reported in a case of PTC (The Cancer Genome Atlas-EM-A3ST-01). EIF1AX exon2 mutations without $H / N / K-R A S$ mutations, which existed in benign lesions, borderline lesions and PTC, but not in FTC, PDTC and ATC, might be endowed as a diagnostic marker.

BRAF V600E mutation was absent in WDT-UMP, which is consistent with previous studies [2, 35]. Recently, Amendoeira et al's review summarized the genotypic abnormalities of NIFTP in several studies. From their reports, $H / N / K-R A S$ mutations were detected in $44.9 \%$ NIFTP cases $(119 / 265)$ and more than half of the previous studies detected no BRAF V600E mutation in NIFTP cases [36]. The research of Kim et al. also showed a similar $H / N / K-R A S$ mutation rate (47\%), and absence of BRAF V600E mutation in their NIFTP series [37]. In the present study, we identified $H / N / K-R A S$ mutations and BRAF mutations respectively in 53 and $0 \%$ of the WDT-UMP cases. The result suggests that WDT-UMP might be genetically similar to non-invasive follicular thyroid neoplasm with papillary-like nuclear features (NIFTP). A previous report identified a relatively low rate of $H / N / K-R A S$ mutations in WDT-UMP [36]. One plausible reason for the difference is that the majority of our WDT-UMP cases had PTC-type nuclear 
alteration, which histologically overlapped with NIFTP. However, given the limitation of our single institution experience, the results need further validation in multicenter studies with large cohorts of patients.

TP53 mutations were not identified in FTA group but in borderline/malignant tumors. In the TP53-mutated WDT-UMP, the tumor size was $4 \mathrm{~cm}$, much larger than the median size of $1.2 \mathrm{~cm}$ in WDT-UMP group. Those suggested a possible subgroup of TP53-mutated tumors, and its potential role in progression to a more aggressive phenotype that has not yet fully manifested in thyroid tumors [37, 38]. Although TP53 mutations were more found in FT-UMP than in FTC, there was no significant difference regarding the prevalence of TP53 mutations. That may result from the small sample size in our cohort. Further studies clarifying the role of TP53 mutations in patient diagnosis and prognostic significance in larger sample size should be organized [37].

The frequency of PAX8-PPARG fusion in our cohort was much lower than $30-60 \%$ noted in reports from other countries [39]. Kunio et al. also detected low frequency of PAX8-PPARG fusion $(1 / 24,4 \%)$ in FTC, suggesting a possible distinct genetic feature in FTC in Japanese patients due to the high iodine intake from a typical Japanese diet [40]. Chinese population has an iodized salt diet, for the iodized salt policy has been implemented by Chinese government since 1995 . It implicates that the demographic background may have an influence on the rate of PAX8-PPARG fusion.

In summary, TERTp, EIF1AX, TSHR, H/N/K-RAS and TP53 mutations may have diagnostic and prognostic potential in follicular thyroid tumors. TERTp mutations may be indicative of poor outcome in FTC patients with minimally invasive/ encapsulated angioinvasive histological features.

\section{Abbreviations}

AFTA: Atypical follicular thyroid adenoma; AJCC: The American Joint Committee on Cancer; ATC: Anaplastic thyroid cancer; COSMIC: The Catalogue Of Somatic Mutations In Cancer; DFS: Disease-free survival; FFPE: Formalin-fixed, paraffin-embedded; FTA: Follicular thyroid adenoma; FTC: Follicular thyroid cancer; FT-UMP: Follicular tumors of uncertain malignant potential; NGS: Next- generation sequencing; PDTC: Poorly differentiated thyroid cancer; PTC: Papillary thyroid cancer; TERTp: TERT promoter; TT-UMP: Thyroid tumors with uncertain malignant potential; WDTUMP: Well-differentiated tumors of uncertain malignant potential; WHO: World Health Organization

\section{Acknowledgements}

The authors would like to thank the laboratory technician, Hong Cheng, for preparing the samples.

\section{Funding}

This research is supported by Chinese Academy of Medical Sciences (CAMS) Initiative for Innovative Medicine (CAMS-12M) (Project No. 2017-I2M-1-001 and 2016-12M-1-002).

\section{Availability of data and materials}

The datasets analysed during the current study are available from the corresponding author on reasonable request.

\section{Authors' contributions}

$\mathrm{ZL}$ and $\mathrm{HW}$ designed the project. $\mathrm{HD}, \mathrm{XL}, \mathrm{HW}, \mathrm{XR}$ and $\mathrm{HZ}$ performed the test. $\mathrm{HD}$ and HW performed the data analysis and finished the paper writing. All authors read and approved the final manuscript.

\section{Ethics approval and consent to participate}

The institutional review board of Peking Union Medical College Hospital approved the study. For this retrospective study formal informed consent is not required.

\section{Consent for publication}

Not applicable.

\section{Competing interests}

The authors declare that they have no competing interests.

\section{Publisher's Note}

Springer Nature remains neutral with regard to jurisdictional claims in published maps and institutional affiliations.

Received: 28 February 2019 Accepted: 18 April 2019

Published online: 10 May 2019

References

1. Cabanillas ME, McFadden DG, Durante C. Thyroid cancer. Lancet. 2016;388: 2783-95. https://doi.org/10.1016/s0140-6736(16)30172-6.

2. Lloyd RV OR KG, Rosai J. 2017 WHO classification of Tumours of endocrine organs WHO/IARC classification of Tumours. 2017; 4th edition, volume 10

3. Cipriani NA, Nagar S, Kaplan SP, et al. Follicular thyroid carcinoma: how have histologic diagnoses changed in the last half-century and what are the prognostic implications? Thyroid. 2015;25:1209-16. https://doi.org/10. 1089/thy.2015.0297.

4. Foukakis T, Gusnanto A, Au AYM, et al. A PCR-based expression signature of malignancy in follicular thyroid tumors. Endocr Relat Cancer. 2007;14:38191. https://doi.org/10.1677/Erc-06-0023.

5. Horn S, Figl A, Rachakonda PS, et al. TERT promoter mutations in familial and sporadic melanoma. Science. 2013;339:959-61. https://doi.org/10.1126/ science.1230062

6. Wang N, Liu T, Sofiadis A, et al. TERT promoter mutation as an early genetic event activating telomerase in follicular thyroid adenoma (FTA) and atypical FTA. Cancer. 2014;120:2965-79. https://doi.org/10.1002/cncr.28800.

7. Melo M, da Rocha AG, Vinagre J, et al. TERT promoter mutations are a major Indicator of poor outcome in differentiated thyroid carcinomas. J Clin Endocr Metab. 2014;99:E754-65. https://doi.org/10.1210/jc.2013-3734.

8. Muzza M, Colombo C, Rossi S, et al. Telomerase in differentiated thyroid cancer: promoter mutations, expression and localization. Mol Cell Endocrinol. 2015;399:288-95. https://doi.org/10.1016/j.mce.2014.10.019.

9. Vinagre J, Almeida A, Populo $\mathrm{H}$, et al. Frequency of TERT promoter mutations in human cancers. Nat Commun. 2013; 4. Artn 2185 10.1038/ Ncomms3185.

10. Liu RY, Xing MH. Diagnostic and prognostic TERT promoter mutations in thyroid fine-needle aspiration biopsy. Endocr Relat Cancer. 2014;21:825-30. https://doi.org/10.1530/Erc-14-0359.

11. Paulsson JO, Mu N, Shabo I, et al. TERT aberrancies: a screening tool for malignancy in follicular thyroid tumours. Endocr Relat Cancer. 2018. https:// doi.org/10.1530/ERC-18-0050

12. Proietti A, Sartori C, Macerola E, et al. Low frequency of TERT promoter mutations in a series of well-differentiated follicular-patterned thyroid neoplasms. Virchows Arch. 2017:471:769-73. https://doi.org/10.1007/s00428017-2236-6.

13. Liu R, Xing M. TERT promoter mutations in thyroid cancer. Endocr Relat Cancer. 2016;23:R143-55. https://doi.org/10.1530/ERC-15-0533.

14. Lancia I, Ibrahimpasic T, Boucai L, et al. Genomic and transcriptomic hallmarks of poorly differentiated and anaplastic thyroid cancers. J Clin Invest. 2016;126:1052-66. https://doi.org/10.1172/Jci85271.

15. Simoes-Pereira J, Moura MM, Marques IJ, et al. The role of EIF1AX in thyroid cancer tumourigenesis and progression. J Endocrinol Investig. 2018. https:// doi.org/10.1007/s40618-018-0919-8.

16. Patrinos GP. DPaAW. Molecular Diagnostics 2017

17. Kwak JY, Kim EK, Chung WY, Moon HJ, Kim MJ, Choi JR. Association of BRAFV600E mutation with poor clinical prognostic factors and US features 
in Korean patients with papillary thyroid microcarcinoma. Radiology. 2009; 253:854-60. https://doi.org/10.1148/radiol.2533090471.

18. Catalogue of somatic mutations in Cancer. https://cancer.sanger.ac.uk/ cosmic/. Accessed July 22018.

19. Tan J, Ong CK, Lim WK, et al. Genomic landscapes of breast fibroepithelial tumors. Nat Genet. 2015;47:1341-5. https://doi.org/10.1038/ng.3409.

20. Nikiforov YE, Carty SE, Chiosea SI, et al. Highly accurate diagnosis of Cancer in thyroid nodules with follicular neoplasm/suspicious for a follicular neoplasm cytology by ThyroSeq v2 next-generation sequencing assay. Cancer. 2014;120:3627-34. https://doi.org/10.1002/cncr.29038.

21. Cerami E, Gao J, Dogrusoz U, et al. The cBio cancer genomics portal: an open platform for exploring multidimensional cancer genomics data. Cancer Discov. 2012;2:401-4. https://doi.org/10.1158/2159-8290.CD-12-0095.

22. Gao J, Aksoy BA, Dogrusoz U, et al. Integrative analysis of complex cancer genomics and clinical profiles using the cBioPortal. Sci Signal. 2013;6:pl1. https://doi.org/10.1126/scisignal.2004088.

23. The catalogue of somatic mutations in Cancer. https://cancer.sanger.ac.uk/ cosmic/gene/analysis?!n=EIF1AX\#variants. Accessed 18 July 2018.

24. cBioPortal for cancer genomics. http://www.cbioportal.org/. Accessed July 42018.

25. Karunamurthy A, Panebianco F, S JH, et al. Prevalence and phenotypic correlations of EIF1AX mutations in thyroid nodules. Endocr Relat Cancer. 2016:23:295-301. https://doi.org/10.1530/ERC-16-0043.

26. Topf MC, Wang ZX, Tuluc M, Pribitkin EA. TERT, HRAS, and EIF1AX mutations in a patient with follicular adenoma. Thyroid. 2018. https://doi.org/10.1089/ thy.2017.0504

27. Topf MC, Wang ZX, Furlong K, Miller JL, Tuluc M, Pribitkin EA. EIF1AX mutation in a patient with Hurthle cell carcinoma. Endocr Pathol. 2018;29: 27-9. https://doi.org/10.1007/s12022-017-9501-8.

28. Sponziello M, Silvestri G, Verrienti A, et al. A novel nonsense EIF1AX mutation identified in a thyroid nodule histologically diagnosed as oncocytic carcinoma. Endocrine. 2018. https://doi.org/10.1007/s12020-0181611-7.

29. Kunstman JW, Juhlin CC, Goh G, et al. Characterization of the mutational landscape of anaplastic thyroid cancer via whole-exome sequencing. Hum Mol Genet. 2015;24:2318-29. https://doi.org/10.1093/hmg/ddu749.

30. Kennichi K. How to handle borderline/precursor thyroid tumors in management of patients with thyroid nodules.; 2018. https://doi.org/10. 21037/gs.2017.08.02

31. Bournaud C, Descotes F, Decaussin-Petrucci M, et al. TERT promoter mutations identify a high-risk group in metastasis-free advanced thyroid carcinoma. Eur J Cancer. 2019;108:41-9. https://doi.org/10.1016/j.ejca.2018.12.003.

32. Song YS, Lim JA, Min HS, et al. Changes in the clinicopathological characteristics and genetic alterations of follicular thyroid cancer. Eur J Endocrinol. 2017;177:465-73. https://doi.org/10.1530/EJE-17-0456.

33. Sohn SY, Park WY, Shin HT, et al. Highly concordant key genetic alterations in primary tumors and matched distant metastases in differentiated thyroid Cancer. Thyroid. 2016;26:672-82. https://doi.org/10.1089/thy.2015.0527.

34. Radkay LA, Chiosea SI, Seethala RR, et al. Thyroid nodules with KRAS mutations are different from nodules with NRAS and HRAS mutations with regard to cytopathologic and histopathologic outcome characteristics. Cancer Cytopathol. 2014;122:873-82. https://doi.org/10.1002/cncy.21474.

35. Liu Z, Zhou G, Nakamura M, et al. Encapsulated follicular thyroid tumor with equivocal nuclear changes, so-called well-differentiated tumor of uncertain malignant potential: a morphological, immunohistochemical, and molecular appraisal. Cancer Sci. 2011;102:288-94. https://doi.org/10.1111/j.1349-7006. 2010.01769.x.

36. Hofman V, Lassalle $\mathrm{S}$, Bonnetaud $\mathrm{C}$, et al. Thyroid tumours of uncertain malignant potential: frequency and diagnostic reproducibility. Virchows Arch. 2009:455:21-33. https://doi.org/10.1007/s00428-009-0798-7.

37. Marotta V, Sciammarella C, Colao A, Faggiano A. Application of molecular biology of differentiated thyroid cancer for clinical prognostication. Endocr Relat Cancer. 2016;23:R499-515. https://doi.org/10.1530/ERC-16-0372.

38. Nikiforov YE, Nikiforova MN, Gnepp DR, Fagin JA. Prevalence of mutations of ras and p53 in benign and malignant thyroid tumors from children exposed to radiation after the Chernobyl nuclear accident. Oncogene. 1996;13:687-93.

39. Yakushina VD, Lerner LV, Lavrov AV. Gene fusions in thyroid Cancer. Thyroid. 2018;28:158-67. https://doi.org/10.1089/thy.2017.0318.

40. Mochizuki K, Kondo T, Oishi N, et al. Low frequency of PAX8-PPARgamma rearrangement in follicular thyroid carcinomas in Japanese patients. Pathol Int. 2015;65:250-3. https://doi.org/10.1111/pin.12270.

Ready to submit your research? Choose BMC and benefit from:

- fast, convenient online submission

- thorough peer review by experienced researchers in your field

- rapid publication on acceptance

- support for research data, including large and complex data types

- gold Open Access which fosters wider collaboration and increased citations

- maximum visibility for your research: over $100 \mathrm{M}$ website views per year

At BMC, research is always in progress.

Learn more biomedcentral.com/submissions 